\title{
Diagnostic accuracy of digital RNA quantification versus real-time PCR for the detection of respiratory syncytial virus in nasopharyngeal aspirates from children with acute respiratory infection
}

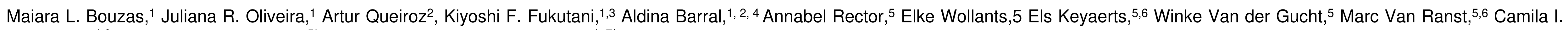
de Oliveira, ${ }^{1,2}$ Johan Van Weyenbergh, ${ }^{*}$ Cristiana M. Nascimento-Carvalho, ${ }^{1,7^{*}}$ and The Acute Respiratory Infection and Wheeze Study Group Phase I and II.

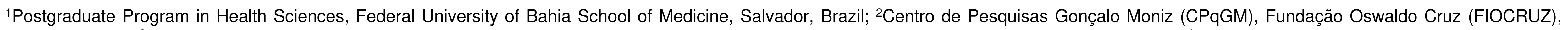

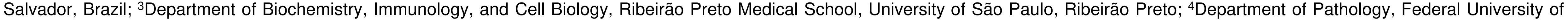

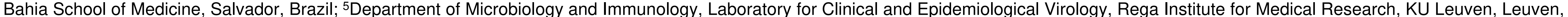

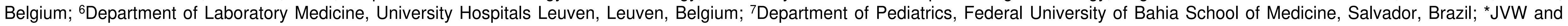
$\mathrm{CMN}-\mathrm{C}$ are both senior authors.

Respiratory syncytial virus (RSV) is one of the most common etiological agents of acute respiratory infections (ARI) among children such as bronchiolitis and pneumonia. ${ }^{1}$ Virusspecific molecular assays such as real-time polymerase chain reaction (RT-PCR) are now considered the gold standard in the diagnosis of viral respiratory tract infections, but simultaneous (multiplex) detection of different pathogens is limited which are considered majors limitations. ${ }^{2} \mathrm{~A}$ multiplex digital method of RNA quantification, nCounter (NanoString Technologies), can overcome this disadvantage and identify, in a single reaction, the presence of different respiratory viruses. ${ }^{3}$ We aimed to evaluate the accuracy of nCounter (Nanostring Technologies) to identify and quantify RSV-A and RSV-B in NPA of children with ARI using real-time PCR as the reference method.

1. Nascimento-Carvalho CM, Cardoso MRA, Barral A et al. Seasonal patterns of viral and bacterial infections among children hospitalized with community-acquired pneumonia in a tropical region. Scand. J. Infect. Dis. 42 (2010) 839-844. 2. Thorburn F, Bennett $S$, Modha $S$ et al. The use of next generation sequencing in the diagnosis and typing of respiratory infections. J. Clin. Virol. 69 (2015) 96-100. 3. Geiss GK, Bumgarner RE, Birditt B et al. Direct multiplexed measurement of gene expression with colorcoded probe pairs. Nat Biotechnol. 2008 Mar;26(3):317-25. Funding

This work was supported by Fundação de Amparo à pesquisa o Estado da Bahia (FAPESB; grant PNX0019/2009). MLB was supported by fellowships from FAPESB. KFF was supported by fellowships from Conselho Nacional de Desenvolvimento Científico e Tecnológico (CNPQ), FAPESB and Fundação de Amparo à Pesquisa do Estado de São Paulo (FAPESP no. 2017/03491-6). JRO was supported by fellowships from FAPESB and Coordenação de Aperfeiçoamento de Pessoal de Nivel Superior (CAPES). AB, $\mathrm{CIO}, \mathrm{CMN}-\mathrm{C}$ are senior investigators from CNPQ. JVW was
supported by FWO (grant G0D6817N).
NPA was collected at enrolment in a prospective cross-sectional study conducted in an Emergency Department from September 2009 to October 2013, in Salvador, Brazil. A quantitative RT-PCR with a subgroup-specific primer and probeset for RSVA and RSV-B was performed in parallel with a customized nCounter probeset containing viral targets in NPA.

Table 1A. Diagnostic accuracy of nCounter to detect RSV-A

\begin{tabular}{|c|c|c|c|c|}
\hline & & & & Total \\
\hline & & Yes & No & \\
\hline & Yes & $55(74.3 \%)$ & $8(1.6 \%)$ & $63(11.3 \%)$ \\
\hline nCounter & No & $19(25.7 \%)$ & $477(98.4 \%)$ & $496(88.7 \%)$ \\
\hline & Total & $74(13.2 \%)$ & $485(86.8 \%)$ & $559(100 \%)$ \\
\hline
\end{tabular}

Table 1B. Diagnostic accuracy of nCounter to detect RSV-B

\begin{tabular}{ccccc}
\hline \multicolumn{4}{c}{ RT-PCR } & Total \\
\hline \multirow{3}{*}{ nCounter } & Yes & $52(77.6 \%)$ & $11(2.2 \%)$ & $63(11.3 \%)$ \\
& No & $15(22.4 \%)$ & $481(97.8 \%)$ & $496(88.7 \%)$ \\
\cline { 2 - 5 } & Total & $67(12.0 \%)$ & $492(88.0 \%)$ & $559(100 \%)$ \\
\hline
\end{tabular}

This study group comprised 559 cases and RSV was detected by RT-PCR in 139 (24.9\%) cases, RSV-A in 74 (13.2\%) cases, and RSV $B$ in $67(12.0 \%)$ cases. Two $(1.4 \%)$ were co infected.
Digital quantification by nCounter, the new diagnostic method, detected RSV in 122 $(21.8 \%)$ samples, RSV-A in 63 (11.3\%) and RSV-B in $63(11.3 \%)$. Co-infections were detected in $4(3.3 \%)$ cases. Interestingly, detection of RSV-A and RSV-B by any method occurred in 158 (28.3\%) cases, indicating both methods might be complementary in detecting the complete RSV epidemic.

The validation of nCounter as a qualitative measure (presence vs. absence) considering RT-PCR as the reference standard is shown in Table 1A for RSV-A and in Table 1B for RSV-B.

Overall, accuracy was $95.2 \%(95 \% \mathrm{Cl}: 93.1 \%$ $96.7 \%$ ) for RSV-A and $95.3 \%$ (95\% Cl:93.3\% 96.9\%) for RSV-B. Moreover, quantification of both RSV-A and RSV-B viral RNA was significantly correlated between nCounter and RT-PCR, as shown in Fig. 1A-B

Again, using RT-PCR as a reference, a significant correlation (Spearman $r=0.44$, $\mathrm{p}=8 \times 10^{-5}$ ) was found in RSV-A-positive samples $(n=74)$, between quantitative detection by nCounter (measured as normalized counts) and RT-PCR (measured as copies $/ \mathrm{ml}$ ). Similarly, in RSV-B RT-PCR positive samples $(n=67), \quad$ a significan correlation (Spearman $r=0.73, \quad p=3 \times 10^{-12}$ ) was found between RSV-B quantitative detection by $n$ Counter and RT-PCR (Fig. 1B

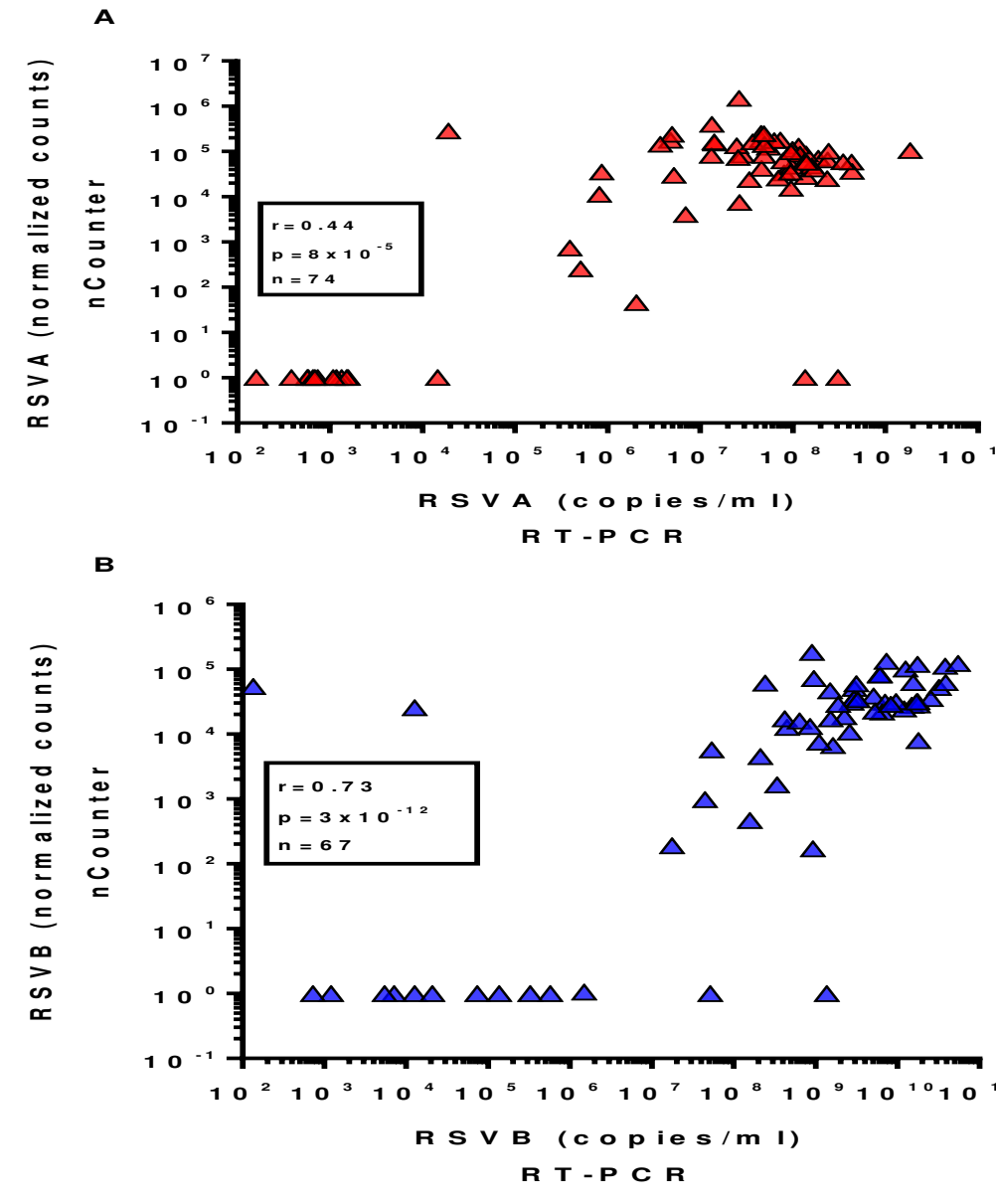

Figure 1: Correlation between RSV-A and RSV$B$ levels quantified by nCounter and Real-Time PCR. (A) In RT-PCR-positive samples $(\mathrm{n}=74)$, a significant correlation (Spearman $\mathrm{r}=0.44, \mathrm{p}=8 \times 10$ 5) was found between RSV-A quantitative detection by nCounter (measured as normalized counts) and RT-PCR (measured as copies/ml). (B) In RT-PCR-positive samples ( $\mathrm{n}=67)$, a significant correlation (Spearman $\mathrm{r}=0.73, \mathrm{p}=3 \times 10-12$ ) was found between RSV-B quantitative detection by nCounter (normalized counts) and RT-PCR (copies $/ \mathrm{ml}$ ).

In conclusion, digital RNA quantification of RSV-A and RSV-B by nCounter is highly accurate (>95\%), using real-time PCR as a reference. Its robustness, high-throughput multiplex capacity and detection of cases undetected by real-time PCR indicate its suitability for large-scale epidemiological studies. 